\title{
Editorial
}

\section{Optimization Theory, Methods, and Applications in Engineering}

\author{
Jung-Fa Tsai, ${ }^{1}$ John Gunnar Carlsson, ${ }^{2}$ Dongdong Ge, ${ }^{3}$ \\ Yi-Chung $\mathrm{Hu}^{4}{ }^{4}$ and Jianming $\mathrm{Shi}^{5}$ \\ ${ }^{1}$ Department of Business Management, National Taipei University of Technology, No. 1, Section 3, \\ Chung-Hsiao East Road, Taipei 10608, Taiwan \\ ${ }^{2}$ Program in Industrial and Systems Engineering, University of Minnesota, 111 Church Street SE, \\ Minneapolis, MN 55455, USA \\ ${ }^{3}$ Antai College of Economics \& Management, Shanghai Jiao Tong University, Room 423, North Building, \\ 535 Fahua Zhen Road, Changning, Shanghai 200052, China \\ ${ }^{4}$ Department of Business Administration, Chung Yuan Christian University, Chung-Li 320, Taiwan \\ ${ }^{5}$ Department of Information and Electronic Engineering, Muroran Institute of Technology, \\ 27-1 Mizumoto-cho, Muroran 050-8585, Japan
}

Correspondence should be addressed to Jung-Fa Tsai, jftsai@ntut.edu.tw

Received 4 September 2012; Accepted 4 September 2012

Copyright (C) 2012 Jung-Fa Tsai et al. This is an open access article distributed under the Creative Commons Attribution License, which permits unrestricted use, distribution, and reproduction in any medium, provided the original work is properly cited.

Over years of development, optimization theory and methods have grown in their ability to handle various practical problems. In light of advances in computing systems, optimization approaches have become one of the most promising techniques for engineering applications. To close the gap between optimization theory and the practice of engineering, this special issue intends to provide the details of recent advances of optimization sciences and promote the applications of optimization methods in engineering. This special issue also provides a forum for researchers and practitioners to review and disseminate quality research work on optimization approaches and their applications in the context of engineering and to identify critical issues for further developments.

The papers accepted in the special issue include original research articles as well as review articles on all aspects of optimization including deterministic approaches, continuous, mixed-integer and discrete optimization, stochastic optimization, particle swarm optimization, neural network, simulated annealing, genetic algorithm, and hybrid methods. Some of the papers are dedicated to the development of advanced optimization methods for direct or indirect use in engineering problems such as network, scheduling, production planning, industrial engineering, and manufacturing systems. Contributions containing computational 
issues, search strategies, and modeling and solution techniques to practical problems such as multicriteria decision making and management information system are also involved. According to the characteristics of the accepted papers, the special issue is organized as the following four parts and each part is composed of several important papers to the part's scope.

\section{Deterministic Optimization}

The paper "Global sufficient optimality conditions for a special cubic minimization problem" by X. Zhang et al. presents some sufficient global optimality conditions for a special cubic minimization problem with box constraints or binary constraints by extending the global subdifferential approach proposed by V. Jeyakumar in 2006. Numerical examples demonstrate that the optimality conditions can effectively be used for identifying global minimizers of the certain nonconvex cubic minimization problem.

Baiqing $\mathrm{Hu}$, Lubin Chang, An Li, and Fangjun Qin propose a comment on "Highly efficient sigma point filter for spacecraft attitude and rate estimation" by Fan and Zeng (2009). In the comment, the geometric simplex sigma points (GSSPs) can be derived from a numerical integration formula of degree 2 and can be generalized for different degrees, that is, the GSSP can be derived through the orthogonal transformation from the basic points set of degree 2 . Moreover, their method can be used to construct more accurate sigma points set for certain dynamic problems.

"Adaptive method for solving optimal control problem with state and control variables" by L. Kahina and A. Mohamed solves the problem of optimal control with state and control variables by adaptive method and technology of linear programming. The obtained results show that it is possible to construct very fast solving algorithms based on the adequate consideration of the dynamic structure of the problem in question.

M. Liu and X. Li propose a filter algorithm with inexact line search for nonlinear programming problems that ensures superlinear local convergence without second order correction steps. In their paper "A filter algorithm with inexact line search" a filter is constructed by employing the norm of the gradient of the Lagrangian function to the infeasibility measure. Numerical experiences show the efficiency of their filter algorithm.

The paper "Predictor-corrector primal-dual interior point method for solving economic dispatch problems: a postoptimization analysis" by A. R. Balbo et al. proposes a predictorcorrector primal-dual interior point method involving line search procedures to solve the economic dispatch problem. Their method applies the Fibonacci search technique in the predictor step and an Armijo line search in the corrector step. The comparative results with other methods described in the literature demonstrate the efficiency of their method.

The paper "An optimal classification method for biological and medical data" by Y.-H. Huang et al. proposes a union of hyper spheres by the mixed-integer nonlinear program to classify biological and medical datasets and a piecewise linearization technique is used to reformulate the nonlinear program to obtain a global optimum. Numerical examples illustrate that the proposed method is computationally more efficient than current methods.

"A review of deterministic optimization methods in engineering and management" by M.$\mathrm{H}$. Lin et al. introduces recent advances in deterministic methods for solving signomial programming problems and mixed-integer nonlinear programming problems. A number of important applications in engineering and management are also reviewed to reveal the usefulness of the optimization methods. 


\section{Heuristic Algorithms}

The paper "Opposition-based barebones particle swarm for constrained nonlinear optimization problems" by H. Wang presents a modified barebones particle swarm optimization to solve constrained nonlinear optimization problems and simulation results show that the presented approach achieves a promising performance.

In "Multithreshold segmentation based on artificial Immune systems," E. Cuevas et al. present an algorithm for multi-threshold segmentation which is based on the artificial immune systems technique. The clonal selection algorithm-based method shows a fast convergence and a low sensitivity to initial conditions and improves complex time-consuming computations commonly required by gradient-based methods.

In "New bounds for ternary covering arrays using a parallel simulated annealing" by $\mathrm{H}$. Avila-George et al. three parallel approaches for simulated annealing: the independent, semiindependent, and cooperative searches are applied to the covering array construction problem. The empirical evidences indicate that cooperative approach offers the best execution times and the same bounds compared to the independent and semiindependent approaches.

In "Applying hierarchical bayesian neural network in failure time prediction," L.-J. Kao and H.-F. Chen apply the Hierarchical Bayesian neural network (HBNN) approach to predict the failure time and utilize the Gibbs sampler of Markov Chain Monte Carlo to estimate statistical model parameters. The results of sensitivity analysis show that HBNN can provide not only the predictive distribution but also the heterogeneous parameter estimates for each path.

In "Applying neural networks to prices prediction of crude oil futures," J. Wei-Shan $\mathrm{Hu}$ et al. attempt to accurately forecast prices of crude oil futures by adopting three popular neural networks methods including the multilayer perceptron, the Elman recurrent neural network, and recurrent fuzzy neural network (RFNN). Experimental results indicate that the use of neural networks to forecast the crude oil futures prices is appropriate and consistent learning is achieved by employing different training times. Moreover, the RFNN outperforms the other two neural networks in forecasting crude oil futures prices.

The paper "Solving the tractor and semi-trailer routing problem based on a heuristic approach" by $\mathrm{H}$. Li et al. develops a heuristic algorithm to solve the tractor and semi-trailer routing problem (TSRP). The computational study shows that their method takes relatively little time to obtain satisfactory solutions.

L. Yao and J. Xu tackle a stone resource assignment problem with the aim of decreasing dust and waste water emissions. In their paper "A stone resource assignment model under the fuzzy environment" a bilevel multiobjective optimization model with fuzzy coefficients is constructed. They design a fuzzy simulation-based improved simulated annealing algorithm (FS-ISA) to search for Pareto optimal solutions. They also present a case study to demonstrate the practicality and efficiency of their model.

"A modified PSO algorithm for minimizing the total costs of resources in MRCPSP" by M. Khalilzadeh et al. introduces a multimode resource-constrained project scheduling problem with finish-to-start precedence relations among project activities, considering renewable and nonrenewable resource costs. The authors formulate this problem as a mixed-integer programming model and present a metaheuristic algorithm based on a modified particle swarm optimization approach. Experimental results reveal the effectiveness and efficiency of their algorithm.

The paper "A nonlinear multiobjective bilevel model for minimum cost network flow problem in a large-scale construction project" by $\mathrm{J}$. Xu et al. deals with a minimum cost network flow problem in a large-scale construction project using a nonlinear multiobjective bilevel model 
with birandom variables. Results and analysis are presented to highlight the performances of the proposed method which is more effective and efficient compared to a genetic algorithm and a simulated annealing algorithm.

"Sparse signal recovery via ECME thresholding pursuits" by $\mathrm{H}$. Song and G. Wang develops ECME thresholding pursuits (EMTPs) for sparse signal recovery. Two effective support detection strategies (hard thresholding and dynamic thresholding) are devised for the sparse signals with components having a fast decaying distribution of nonzero components. The experimental studies are presented to demonstrate that EMTP offers an appealing alternative to state-of-the-art algorithms for sparse signal recovery.

\section{Hybrid Methods}

The paper "Solving constrained global optimization problems by using hybrid evolutionary computing and artificial life approaches" by J.-Y. Wu presents a hybrid real-coded genetic algorithm with a particle swarm optimization (PSO) algorithm and a hybrid artificial immune algorithm with a PSO algorithm for solving 13 constrained global optimization problems, including 6 nonlinear programming and 6 generalized polynomial programming optimization problems. Experimental results indicate that the proposed algorithms converge to a global optimum solution.

In "Combining diffusion and grey models based on evolutionary optimization algorithms to forecast motherboard shipments," F.-K. Wang et al. develop a combined model based on the rolling Grey model and the Bass diffusion model to forecast motherboard shipments. The results indicate that the proposed model using a hybrid algorithm outperforms other methods for the fitting and forecasting processes in terms of mean absolute percentage error.

The paper "Hybrid optimization approach for the design of mechanisms using a new error estimator" by A. Sedano et al. proposes a hybrid optimization algorithm that combines the advantages of both stochastic and deterministic approaches to the design of mechanisms. Two engineering applications, a four-bar linkage and an injection machine, are presented to demonstrate the accuracy, robustness and efficiency of their proposed approach.

"A Hybrid genetic algorithm for the multiple crossdocks problem" by Z. Miao et al. considers multiple crossdocks problem through fixed transportation schedules with time windows, capacity, and penalty. The authors prove that the problem is NP-hard in the strong sense and develop a hybrid genetic algorithm integrating greedy technique and variable neighborhood search method to solve the problem effectively and efficiently.

"A new hybrid Nelder-Mead Particle swarm optimization for Coordination optimization of directional overcurrent relays" by A. Liu and M.-T. Yang proposes a new hybrid Nelder-Mead simplex search method and particle swarm optimization (proposed NM-PSO) algorithm to solve the directional overcurrent relays coordination optimization problem. The findings demonstrate that the performance of the proposed NM-PSO is better than that of PSO and original NM-PSO in terms of computation speed, rate of convergence, and feasibility.

"A VNS metaheuristic with stochastic steps for Max 3-cut and Max 3-section" by A.-f. Ling proposes a local search algorithm and a variable neighborhood global search algorithm with two stochastic search steps to obtain the global solution by establishing a neighborhood structure of the Max 3-cut problem. Numerical results show that the proposed heuristic algorithm can obtain efficiently the high-quality solutions and has the better numerical performance than 0.836-approximate algorithm for the NP-Hard Max 3-cut and Max 3-section problems. 
The paper "Dynamic programming and heuristic for stochastic uncapacitated lot-sizing problems with incremental quantity discount" by Y. Zhanga et al. considers the stochastic uncapacitated lot-sizing problems with incremental quantity discount where the uncertain parameters are supposed to evolve as discrete time stochastic processes. The obtained results reveal that the presented algorithm outperforms the commercial solver CPLEX and other heuristics in both quality of solution and run time.

"A hybrid algorithm based on ACO and PSO for capacitated vehicle routing problems" by Y. Kao et al. proposes a new hybrid algorithm based on two main swarm intelligence approaches, ant colony optimization (ACO) and particle swarm optimization (PSO), for solving capacitated vehicle routing problems. Computational results show that the performance of the proposed method is competitive in terms of solution quality when compared with existing ACO-and PSO-based approaches.

\section{Challenging Applications}

The paper "Goal-programming-driven genetic algorithm model for wireless access point deployment optimization" by C.-S. Wang and C.-T. Chang proposes a method that integrates a goalprogramming-driven model and a genetic algorithm to resolve the multiple objectives appropriate wireless access point deployment problem. Three experiment results demonstrate the utility and stability of the proposed method.

S.-Y. Lin et al. paper "A cost-effective planning graph approach for large-scale web service composition" proposes a novel cost-effective Web service composition mechanism, utilizes planning graph based on backward search algorithm to find multiple feasible solutions, and recommends a best composition solution according to the lowest service cost.

In "A fuzzy dropper for proportional loss rate differentiation under wireless network with a multi-state channel," Y.-C. Szu proposes a novel packet dropper for fuzzy controlling the proportional loss rate differentiation in a wireless network with multi-state channel. Simulation results reveal that the fuzzy proportional loss rate dropper does achieve accurate loss rate proportion, lower queuing delay and loss rate, and higher throughput, compared with other methods in the wireless environment.

The paper "Evaluating the performance of taiwan homestay using analytic network process" by Y.-C. Hu and J.-H. Wang develops and constructs a set of evaluation indicators tailormade for homestay sector through discussion of literatures and interviewing experts so that the evaluation framework would be more comprehensive and more practical.

The paper "Improved degree search algorithms in unstructured P2P networks" by G. Liu et al. proposes two memory function degree search algorithms: memory function maximum degree algorithm and memory function preference degree algorithm and studies their performance including the search success rate and the search message quantity in scale-free networks, random graph networks, and small-world networks.

The paper "Variable neighborhood search for parallel machines scheduling problem with step-deteriorating jobs" by W. Cheng et al. studies a scheduling problem of minimizing the total completion time on identical parallel machines where the processing time of a job is a step function of its starting time and a deteriorating date that is individual to all jobs. The computational results show that the proposed approaches obtain near-optimal solutions in a reasonable computational time even for large-sized problems.

The paper "Solving packing problems by a distributed global optimization algorithm" by N.-Z. Hu et al. develops a novel method to convert the nonlinear objective function in a packing program into an increasing function with a single variable and two fixed parameters. 
The transformed linear program is then decomposed into several subproblems by specifying various parameter values, which is solvable simultaneously by a distributed computation algorithm to obtain a global optimum.

The paper "A hybrid network model to extract key criteria and its application for brand equity evaluation" by C.-Y. Chen and C.-W. Li develops a hybrid model based on social network analysis and maximum mean deentropy algorithms for extracting evaluation criteria which considers substitutions between the criteria. The effectiveness and feasibility of the hybrid model are demonstrated by examples of evaluating brand equity.

The paper "Mixed mortar element method for $P_{1}^{N C} / P_{0}$ element and its multigrid method for the incompressible stokes problem" by Y. Jiang and J. Chen discusses a mortar-type $P_{1}^{N C} / P_{0}$ element method for the incompressible Stokes problem. The inf-sup condition and the optimal error estimate are proved. The study also proposes a W-cycle multigrid for solving the discrete problem and demonstrates the optimal convergence of the multigrid method.

In "Quality improvement and robust design methods to a pharmaceutical research and development" by B. R. Cho and S. Shin, new robust design experimental and optimization models for time-oriented data are applied to the pharmaceutical production research and development. Compared to the traditional Taguchi optimization model, the proposed experimental methodology is particularly useful for experiments with time-oriented pharmaceutical characteristics.

The paper "A novel method for technology forecasting and developing RED strategy of building integrated photovoltaic technology industry" by Y.-J. Chiu and T.-M. Ying proposes a hybrid approach to explore the life cycle of building-integrated photovoltaic (BIPV) technology and develop the R\&D strategy of related industries. The proposed approach comprises patent analysis, logistic growth model analysis, and patent matrix map analysis. The authors also provide three-dimensional matrix of degree of protection, R\&D capability, and benefit creation to select R\&D strategies for BIPV industry.

J.-H. Lin et al. propose a systematic method to analyze student recruitment numbers for future needs, based on the concept of material resource planning (MRP). In their paper "The number of students needed for undeclared programs at a college from the supply-chain viewpoint," the relationship between a curricular structure tree and the associated commonalities is studied and a quantified model of commonality and recruitment planning for appropriate curriculum design is proposed. The authors use two simple examples to illustrate the implementation of MRP in analysis of the replenishment levels in an education system.

The paper "A selection approach for optimized problem-solving process by grey relational utility model and multi-criteria decision analysis" by C.-K. Ke and M.-Y. Wu proposes an approach to assist workers in determining the optimal selection order of candidate actions based on a Grey relational utility model and a multi-criteria decision analysis. Experimental results from analyzing a high-tech company's knowledge base log demonstrate that their selection approach is effective.

M.-H. Lin and H.-J. Hsu addresses an incentive pricing problem for relaying services in multi-hop cellular networks. In their paper "Optimal incentive pricing on relaying services for maximizing connection availability in multi-hop cellular networks" a mathematical programming model is constructed and then solved to determine an optimal incentive price for each intermediate node providing relaying services. The computational results demonstrate that their proposed approach maximizes connection availability of the networks compared to fixed-rate or location-based methods.

"A two stage dea to analyze the effect of entrance deregulation on iranian insurers: a robust approach" by S. G. J. Naini et al. analyzes technical efficiency for Iranian insurance companies 
between 2003 and 2010, a period that insurers experienced intense volatility due to the entrance deregulation of the market by two-stage data envelopment analysis. The major results show that ownership type and failure to meet the risk management rules are the main drivers of efficiency.

"A label correcting algorithm for partial disassembly sequences in the production planning for end-of-life products" by P.-F. Tsai investigates a single period partial disassembly optimization problem to generate an optimal disassembly sequence in product recovery of the end-of-life products. The study presents a heuristic procedure that utilizes a label correcting algorithm which is a polynomial-time algorithm to find a solution. Numerical examples are also used to demonstrate the effectiveness of the proposed solution procedure.

Finally, the guest editors would like to thank the authors for their contributions, the referees for their time and energy in reviewing the paper, and all the staff of Hindawi involved in the preparation of this special issue for their support to publish the articles related to this subject. Without their cooperation, it would have not been possible to edit this special issue.

Jung-Fa Tsai

John Gunnar Carlsson

Dongdong Ge

Yi-Chung $\mathrm{Hu}$

Jianming Shi 


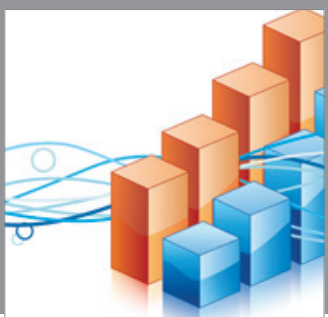

Advances in

Operations Research

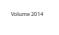

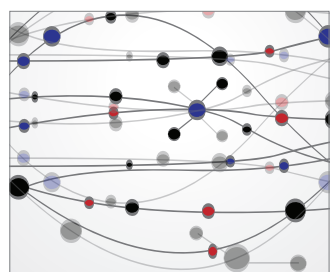

\section{The Scientific} World Journal
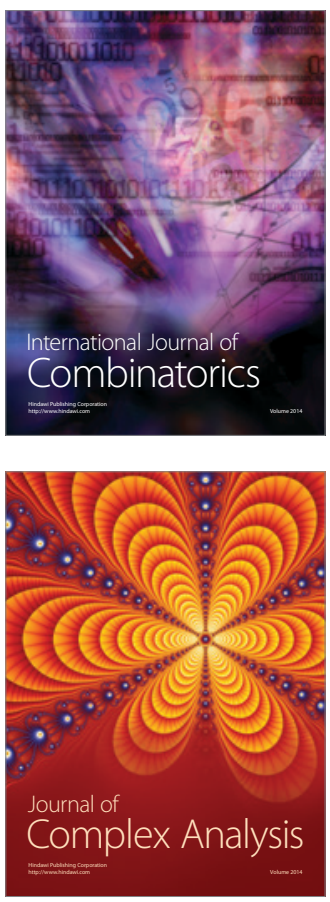

International Journal of

Mathematics and

Mathematical

Sciences
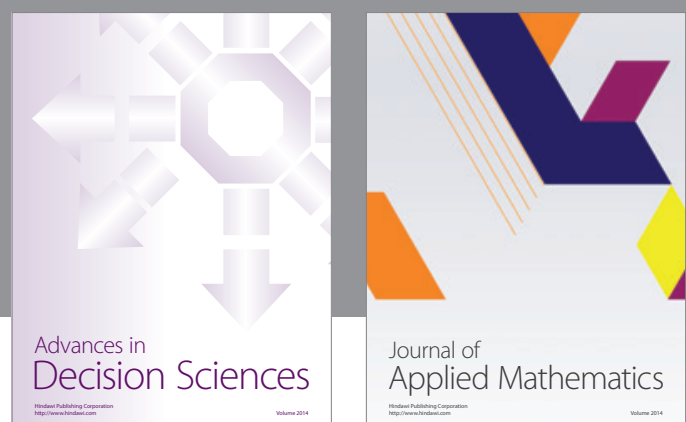

Journal of

Applied Mathematics
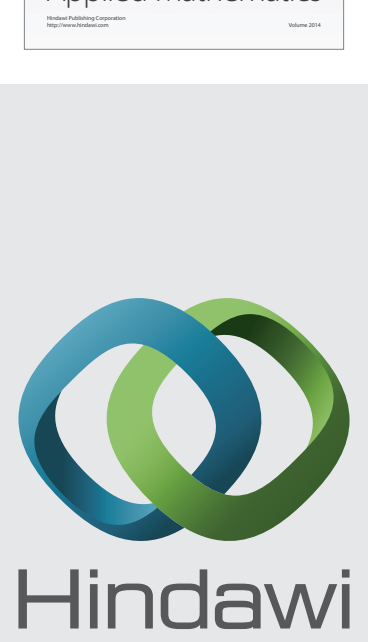

Submit your manuscripts at http://www.hindawi.com
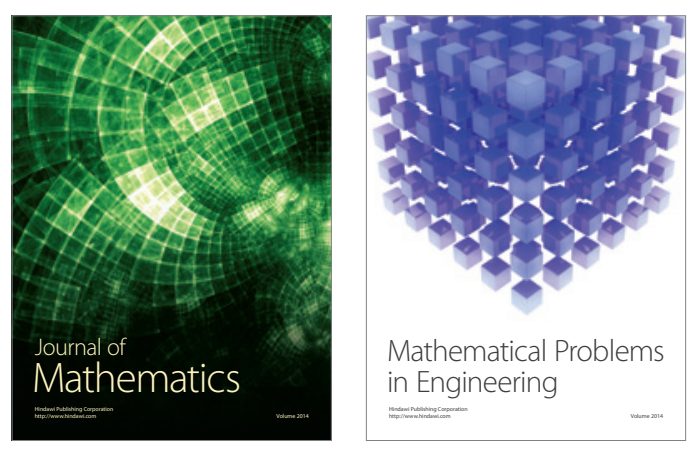

Mathematical Problems in Engineering
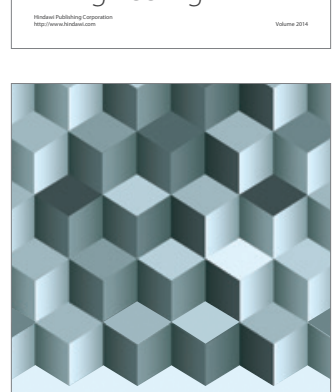

Journal of

Function Spaces
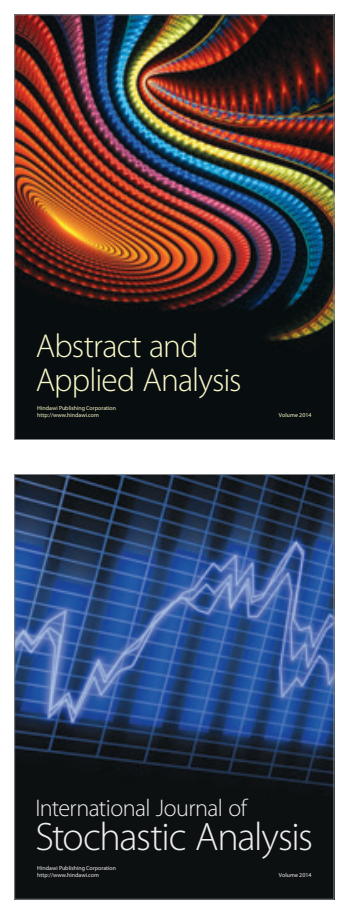

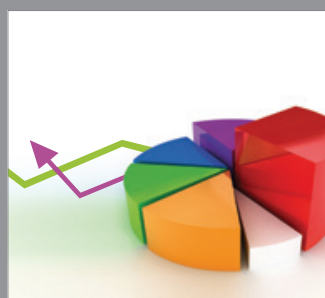

ournal of

Probability and Statistics

Promensencen
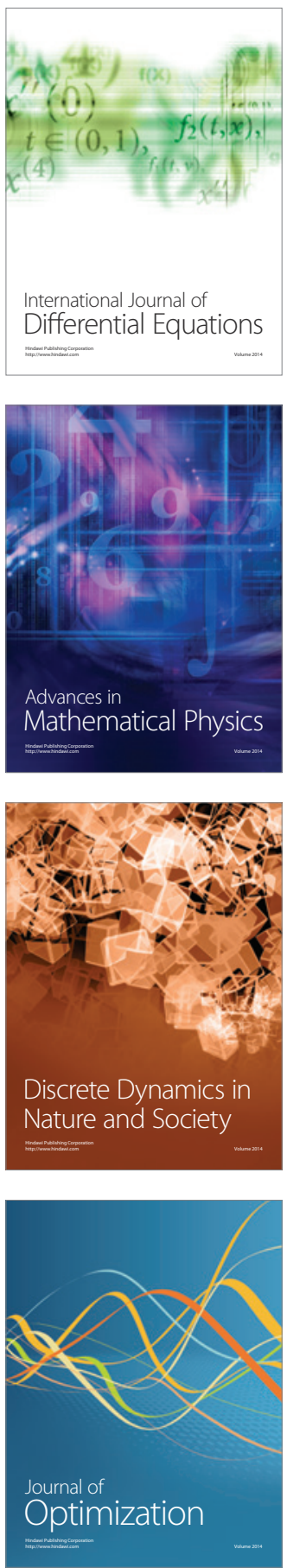